\title{
Clark and Shackel on the Two-Envelope Paradox*
}

\author{
Christopher J. G. Meacham \\ Jonathan Weisberg \\ Published in Mind, 112 (2003): 685-689.
}

Clark and Shackel (2000) have recently argued that previous attempts to resolve the two-envelope paradox fail, and that we must look to symmetries of the relevant expected-value calculations for a solution. Clark and Shackel also argue for a novel solution to the peeking case, a variant of the twoenvelope scenario in which you are allowed to look in your envelope before deciding whether or not to swap. Whatever the merits of these solutions, they go beyond accepted decision theory, even contradicting it in the peeking case. Thus if we are to take their solutions seriously, we must understand Clark and Shackel to be proposing a revision of standard decision theory. Understood as such, we will argue, their proposal is both implausible and unnecessary.

In the version of the two envelope paradox they give, you pick one of two sealed envelopes - let $A$ be the one you pick, and $B$ be the other - each containing some natural power of 2 in dollars. You know that one of the envelopes has twice as much money in it as the other, and you further know that the probability of each possibility is: $p_{0}=p\left(A=2^{0}, B=2^{1}\right)=p(A=$ $\left.2^{1}, B=2^{0}\right)=1 / 12$ and $p_{n+1}=p\left(A=2^{n}, B=2^{n+1}\right)=p\left(A=2^{n+1}, B=\right.$ $\left.2^{n}\right)=1 / 2\left(p_{n}+(1 / 4)^{n}\right), n \geq 1$. You are then offered the opportunity to swap envelopes. Should you?

Standard decision theory tells us to maximize expected utility $(E U)$, where the expected utility of an act is the sum of the products of the probability and utility for all possible outcomes. If there are an infinite number of possibilities over which to sum, the $E U$ is the value to which the series

\footnotetext{
*We would like to thank Frank Arntzenius for his comments on an earlier draft of this paper, and Nicholas Shackel for a helpful clarification of his position.
} 
converges absolutely; if the series does not converge absolutely, the $E U$ is undefined. ${ }^{1}$ In this case the utility of each possibility is the amount of money gained or lost by swapping given a particular pair of values for $A$ and $B .^{2}$ Thus the $E U$ of swapping would be the expectation of $B-A, E(B-A)$, except that this sum does not converge absolutely; given appropriate rearrangements, the sum can be made to converge to any value. So decision theory does not rank swapping against sticking.

Clark and Shackel give this result surprisingly little consideration, dismissing it in an appendix on the grounds that 'expectations for which the corresponding series has no defined sum are themselves undefined and need not be considered.' (Clark and Shackel 2000, p. 435). Instead, they consider three other expectations whose infinite sums do converge absolutely: $E(E(B \mid A)-A), E(B-E(A \mid B))$, and $E(E(B-A \mid A+B))$. But these are not expressions for the $E U$ of swapping given the standard definition of $E U$. Instead of each term in the series corresponding to a possible outcome, these series have as terms the sum of the utility and probability of two possible outcomes. By employing these average expectations, Clark and Shackel go beyond standard decision theory, and become responsible for the formulation and justification of a new theory in which average expectations play a role.

The sums of these three series converge to different values, and Clark and Shackel argue that only $E(E(B-A \mid A+B))$ is correct. They take the symmetric features of the two-envelope game to require that the partial sums of the 'correct' series include the $E U$ contribution of the possibility ( $A=$ $\left.2^{n+1}, B=2^{n}\right)$ in the same term as the possibility $\left(A=2^{n}, B=2^{n+1}\right)$. Since only $E(E(B-A \mid A+B))$, whose partial sums equal and thus converge to 0 , satisfies this constraint, Clark and Shackel claim that the paradox is resolved. Their symmetry constraint straightforwardly entails that the partial sums will equal and thus converge to 0 , however, since the $E U$ contributions of $\left(A=2^{n+1}, B=2^{n}\right)$ and $\left(A=2^{n}, B=2^{n+1}\right)$ always add up to 0 . So their argument hangs on whether or not the symmetry constraint is reasonable. Is it?

We are skeptical, for two reasons. First, the argument Clark and Shackel provide in favor of the constraint would not convince anyone who did not already agree with their conclusion, such as someone who thought $E(E(B \mid A)-$

\footnotetext{
${ }^{1}$ The sum of a sequence $U_{0}, U_{1}, U_{2}, \ldots$ absolutely converges iff $\sum\left|U_{n}\right|$ converges. If a sum $\sum U_{n}$ absolutely converges to $s$, then any sum $\sum V_{n}$ obtained by rearranging the terms of $\sum U_{n}$ will also converge to $s$.

${ }^{2}$ For convenience we assume that utility is linear in dollars.
} 
$A$ ) or $E(B-E(A \mid B))$ was correct. They tell us that:

What distinguishes $E(E(B-A \mid A+B))$ as correct is that only in this case do the partial sums of the expectations properly respect the set-up, namely that, wherever you can have $2^{n}$ in your envelope and twice as much in the other, you can, with equal probability, have $2^{n+1}$ in yours and half as much in the other, and vice versa... only $E(E(B-A \mid A+B))$, which gives zero gain [for each partial sum], respects the symmetry of the set-up for every finite partial sum. (Clark and Shackel 2000, p. 427)

All parties will agree that the infinite sums should respect the symmetry of the set-up, and it follows that for each contribution of a possible outcome in the infinite sum there is an opposite contribution made by the inverse possible outcome. But this entails nothing about what the partial sums need to be like, and does nothing to pick out $E(E(B-A \mid A+B))$ over $E(E(B \mid A)-A)$ or $E(B-E(A \mid B))$. Clark and Shackel are appealing to a further intuition: that, given the setup, the contributions of matched possibilities should be considered together in partial sums. This intuition will not be shared by the proponents of $E(E(B \mid A)-A)$ and $E(B-E(A \mid B))$, however, since how the contributions should be partitioned is precisely what the disagreement is about.

Second, their use of symmetry is puzzling given the project they lay out. Clark and Shackel hope to use the symmetry of the setup to show that the envelopes are equipreferable. But the intuition behind their symmetry constraint just is the intuition that, because the probabilities and utilities are symmetrically distributed, the envelopes ought to be treated identically. And this intuition entails that swapping and sticking are equipreferable. So why do Clark and Shackel go on to apply this intuition to the calculation of average expectations? To us it seems that their strategy adds an unmotivated mathematical epicycle with no explanatory gain.

Let's move on to the peeking case. Unlike the standard case, here you are allowed to peek in your envelope before deciding whether or not to swap. Supposing you find $2^{n}$ dollars, what should you do? According to standard decision theory the relevant expectation is $E\left(B-A \mid A=2^{n}\right)$, which is now given by a finite, two-term sum since only two possibilities, $B=2^{n+1}$ and $B=2^{n-1}$, can obtain. Moreover this sum will be positive, so decision theory tells you to swap. Clark and Shackel, however, argue that swapping and 
sticking are equipreferable (unless $A=1$, in which case $B$ is sure to contain 2 dollars). ${ }^{3}$ Why?

It might seem that we are talking about a situation in which you have different information so that the considerations of [the no-peeking case] do not apply. But this is incorrect. Although if you looked in the envelope you would have different information, whether swapping is better on average is unaffected by that knowledge. (Clark and Shackel 2000, p. 429)

The argument seems to use the assumption that in the no-peeking case swapping has a zero expected gain, to deduce that one should not swap in the peeking case.

Now, we have disputed the claim that there is any well-defined expected gain for the no-peeking case, since $E(B-A)$ is not defined there, so the premise of this argument is dubious. But the argument is a non sequitur as well. What does the expected gain of the no-peeking case have to do with the expected gain of the peeking case? Clark and Shackel argue that, since a policy of swapping would not increase your winnings in repeated peeking games where the probability that $A=2^{n}$ on a given trial is $p_{n}+p_{n-1}$, it follows that swapping is not a beneficial policy in any given case where you know the value of $A$.

But this 'on average' heuristic, where the value of $A$ is not fixed, is irrelevant to the peeking case since the value of $A$ is known. Furthermore, it is misleading to speak of the $E U$ of repeated trials, since in the peeking case the question is whether or not one should swap in a particular case, given that one has seen a particular amount in envelope $A$. Indeed, with some probability distributions it will be the case that the $E U$ of switching is positive for some values of $A$ and negative for others. This makes it especially clear that one's decision regarding whether or not to swap in the peeking case should be determined by the $E U$ of swapping for a particular value of $A$, not on whether the $E U$ of swapping is better 'on average' over repeated trials.

Of course this point is bound to raise the old worry that if swapping is a good idea regardless of the value of $A$, it must be a good idea to swap even if one does not know the value of $A$. Thus one should swap in the nopeeking case. But this reasoning implicitly appeals to the principle that, if

\footnotetext{
${ }^{3}$ While this position is not entirely explicit in Clark and Shackel's The Two-Envelope Paradox, they have confirmed this reading in personal correspondence.
} 
$E(X \mid Z)>E(Y \mid Z)$ for all values of $Z$, then $E(X)>E(Y)$, which Chalmers (2002) has shown to be erroneous. ${ }^{4}$ There is no inconsistency in maintaining that swapping is unhelpful in the no-peeking case but beneficial in the peeking case, and hence no need to follow Clark and Shackel in rejecting standard decision theory.

\section{References}

Arntzenius, F., A. Elga, and J. Hawthorne: manuscript.

Chalmers, David 2002: 'The St. Petersburg Two-Envelope Paradox'. Analysis, 62, pp. 155-57.

Clark, M. and N. Shackel 2000: 'The Two-Envelope Paradox'. Mind, 109, pp. 415-42.

\footnotetext{
${ }^{4}$ That is, let $X=B-A, Y=0$, and $Z=A$. Then inferring from the peeking case that swapping is a good idea in the no-peeking case amounts to using Chalmers' principle to infer $E(B-A)>0$ from the fact that $E\left(B-A \mid A=2^{n}\right)>0$ for all natural $n$. For further discussion and generalization of this diagnosis, see also Arntzenius and Hawthorne (manuscript).
} 